\title{
Crowd flow forecast based on SOM neural network in application of energy-saving lighting
}

\author{
Jun-Feng $\mathrm{Xu}$ and $\mathrm{Li}-\mathrm{Xin} \mathrm{Ma}^{\dagger}$ \\ University of Shanghai for Science and Technology \\ Shanghai, 200093 China \\ E-mail:xiumuqiao@126.com
}

\begin{abstract}
Generally the common lights-control is set in the mode of preset time control, which cannot be adjusted and would not meet the requirements of fine and smart control, so the plug-in of Artificial intelligence algorithms can make a big change of common made. SOM (selforganizing feature map network) is used for flow data feature extraction and classification, and the results are used as inputs of BP network to make a short-term forecast, then combined with the intensity of illumination requirements to output the different rank which is as the same level of flow data, at the end the comparison with traditional way is made in terms of energy saving to prove the advantage of this method of paper. The experimental results show that flow data forecast based on the SOM combined with BP neural network prediction of short-term is more reliable than original BP network, has a better effect in energy-saving, and has provided the new energy conservation plan for the lighting system

Keyword: Artificial intelligence; Energy-saving; SOM network; Lighting-system
\end{abstract}

\section{Introduction}

Up to now, the traditional lighting system which wastes electrical energy, mainly displays that the street light all maintains the entire brightness in the entire illumination time interval, has not rested on the concrete time, the place as well as the stream of people quantity, does not have the time sharing section adjustment in light brightness. And the power loss of traditional street light is big, also the efficiency is low, simultaneously its diameter is thick to be laid down, the cost is high, moreover brightness controllability is bad, its brightness cannot respond the change rapidly. So the traditional lighting system cannot meet the requirements of public lighting design standard.

With Artificial intelligent methods in various fields widely used as well as the amount, method used in energy-efficient lighting is the general trend. Smart mature neural networks have been applied to electric load forecasting, and for regulation of the electric power industry has a significant effect. The SOM neural combined with the BP (Back Propagation) neural network is used for short-term flow forecast of crowd in this paper, and the results of forecast would be used to 
adjust the light intensity of the lighting to achieve energy-saving purpose and effect [1].

\section{Forecasting Method of Crowd Flow}

\subsection{Self-organizing feature map network}

SOM (self-organizing feature map network), simulates the properties of neurons which are the lateral inhibition, self-organizing, refers to one kind of algorithm which has the advantages of characteristic cluster, the high Uygur visualization and non-teacher studies [2-3]. The network structure is shown in Figure.1.

Fig.1.The structure of SOM

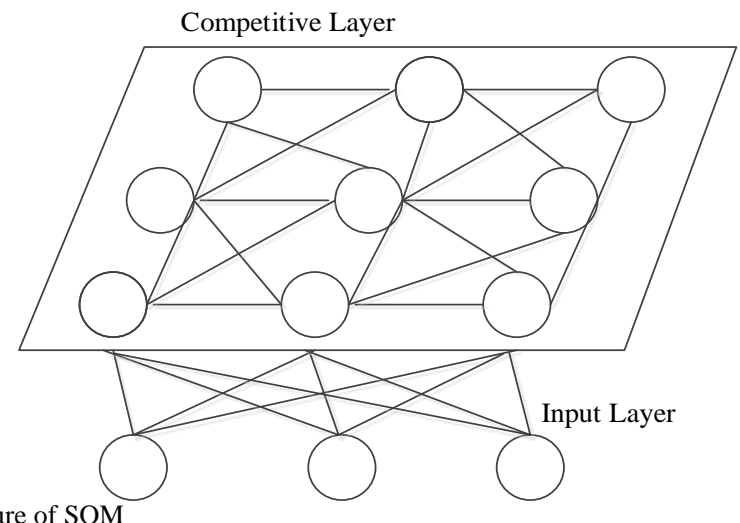

The algorithm flow is as follows [4-5],

(i) Initialization: Take the $[0,1]$ range of random values as neural network connection weights $W_{i j}$, initial field $N_{c}(0)$ is set to a large extent, and network learning rate $\eta(0), 0<\eta(0)<1$; number of iterations is $\mathrm{T}$, then normalization as Eq. (1-2).

$$
\begin{aligned}
& \overline{X_{k}}=\frac{X_{k}}{\left\|X_{k}\right\|}=\frac{\left(X_{1 k}, X_{2 k}, \ldots \ldots X_{n k}\right)}{\sqrt{\left(X_{1 k}\right)^{2}+\ldots+\left(X_{i j}\right)^{2}+\ldots+\left(X_{n k}\right)^{2}}} . \\
& \overline{W_{J}}=\frac{W_{j}}{\left\|W_{j}\right\|}=\frac{\left(W_{1 j}, W_{2 j}, \ldots \ldots W_{n j}\right)}{\sqrt{\left(W_{1 j}\right)^{2}+\ldots+\left(W_{i j}\right)^{2}+\ldots+\left(W_{n j}\right)^{2}}} .
\end{aligned}
$$

In Eq. (1-2), $X_{k}$ is $\mathrm{n}$ dimensional, $k=1,2, \ldots . . n ; W_{j}$ is $\mathrm{n}$ dimensional too, $j=1,2, \ldots . ., m$.

(ii) Accept input: Take any input $X_{k}$ from the input layer of the network, as the input vector.

(iii) Looking for winning node: The neuron $c$ is selected as the winning node, which has the minimum Euclidean distance between $\overline{X_{k}}$ (input vector) and 
$\bar{W}_{J}$ (connection weight vector) as Eq. (3).

$$
\left\|X_{k}-W_{c}\right\|=\min \left\{d_{j k}\right\}
$$

(iv) Parameter adjustment: The winning node $c$ from above and the weight value of all nodes of $c$ 's filed are adjusted as Eq. (4).

$$
\begin{gathered}
W_{i j}(t+1)=W_{i j}(t)+\eta(t) h_{c, j}(t)\left(X_{i}-W_{i j}(t)\right) \\
h_{c, j}(t)=\exp \left(-\frac{d_{c j}{ }^{2}}{2 r^{2}(t)}\right)
\end{gathered}
$$

And $0<\eta(t)<1, \eta(t)$ is the gain function; $h_{c, j}(t)$ is the field function as Eq. (5), $d_{c j}$ means the distance between the winning node $c$ and the output neurons which are activated in any field.

(v) Update the both functions of gain and field: Adjustment rules is as shown in the following Eq. (6).

$$
\begin{gathered}
\left.\mathrm{r}(t+1)=\operatorname{INT}(r(t)-1) \times\left(1-\frac{t}{T}\right)\right)+1 \\
\eta(t+1)=\eta(t)-\frac{\eta(0)}{T}
\end{gathered}
$$

(vi) Circulation study: The other input mode, in turn, repeat from the step (iii), until all the input mode study is completed.

(vii) End: Make $\mathrm{T}=\mathrm{t}+1$, restart from the step (ii), until the $\mathrm{t}=\mathrm{T}$ or the network convergence.

\subsection{The flow prediction based on the combination of SOM and BP}

The crowd flow has the characteristics of nonlinear and randomness, its volatility is caused by many factors. This flow chart of the prediction method is shown in Figure.2.

SOM algorithm is used for feature extraction and classification groups of historical flow data, the same type which is similar to the flow of data is elected as the input sample of BP network to predict the flow data, through the establishment of BP neural network prediction [6-8]. 


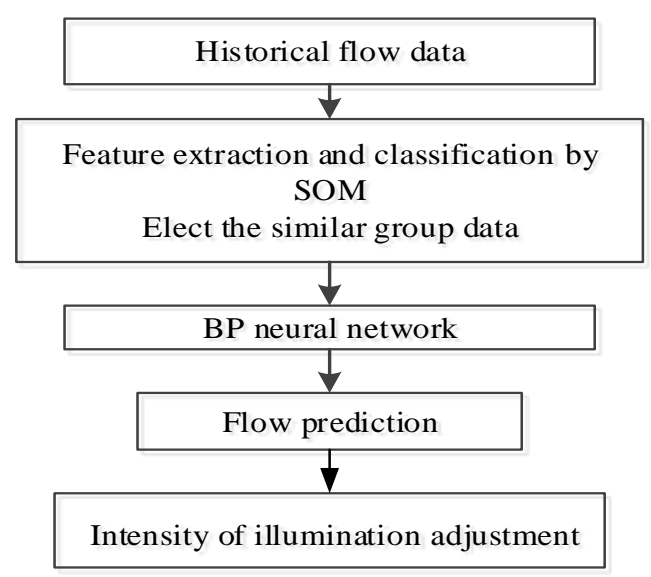

Fig.2. Flow chart of crowd flow prediction based on SOM-BP

\section{Simulation}

Take the crowd flow daily data (12:00-21:00) of a mall from Oct.1 to Oct.30 in 2009 as the input to carry on the cluster, and then make a flow data prediction of Oct.31 by data from above. The data clustering is simulated by MATLAB, the study number of which is 500 times and the results would be put in 3 groups [9]. The clustering result is shown in Table 1.

Table 1. Clustering results

\begin{tabular}{cc}
\hline $\begin{array}{c}\text { Output } \\
\text { neural }\end{array}$ & Clustering results \\
\hline 1 & $1,2,3,4,5$ \\
2 & 7,11 \\
3 & $6,8,9,10,12,13$ \\
\hline
\end{tabular}

The data after clustering is used as input of BP neural network model to predict, the group of data which has the minimum value of error would be elected as the results of prediction. The data contrast chart of flow prediction of Oct.31 is shown in Table 2. APE means the relative error.

The contrast results show that, because the cluster analysis to the historical load data has been carried on before the prediction, then the data as input of prediction has the daily patterns of high similarity to the day flow data predicted. The forecast result accuracy is high than the way in BP only. The Average relative error is $8.98 \%$, less than $23.06 \%$ of BP only prediction. It proves that the SOMBP method can improve the forecast reliability, then make light intensity of lighting adjustment more intelligent and smarter. 
Table 2. Flow prediction data contrast of Oct.31

\begin{tabular}{|c|c|c|c|c|c|}
\hline \multirow{2}{*}{ Time } & \multirow{2}{*}{$\begin{array}{l}\text { Actual } \\
\text { value }\end{array}$} & \multicolumn{2}{|l|}{$\begin{array}{l}\text { BP only } \\
\text { prediction }\end{array}$} & \multicolumn{2}{|l|}{$\begin{array}{l}\text { SOM-BP } \\
\text { prediction }\end{array}$} \\
\hline & & $\begin{array}{l}\text { Predictive } \\
\text { value }\end{array}$ & APE & $\begin{array}{l}\text { Predictive } \\
\text { value }\end{array}$ & APE \\
\hline 12:00 & 444 & 653.8 & 47.26 & 556.8 & 25.4 \\
\hline 13:00 & 1184 & 1051.3 & 11.21 & 1133.6 & 4.25 \\
\hline 14:00 & 1520 & 1982.0 & 30.34 & 1313.8 & 13.57 \\
\hline 15:00 & 1608 & 1291.7 & 19.67 & 1569.6 & 2.39 \\
\hline 16:00 & 1908 & 1765.1 & 7.49 & 1891.0 & 0.89 \\
\hline $17: 00$ & 2128 & 2066.4 & 2.89 & 2106.2 & 1.03 \\
\hline 18:00 & 1032 & 642.1 & 37.78 & 1168.9 & 13.26 \\
\hline 19:00 & 1048 & 716.4 & 31.68 & 1194.8 & 14.01 \\
\hline 20:00 & 1600 & 1347.5 & 15.78 & 1708.0 & 6.75 \\
\hline
\end{tabular}

On this basis, lighting light intensity output is combined with the flow of data, and different crowd flow situations were given different ranks light output [10]. On the premise of meeting lighting needs, according to the rules that output intensity is proportional to the power loss, the proportion of conserving energy on the basis of crowd flow forecast is approximately $12 \%$, compared with the traditional regulation way. The illumination intensity output contrast chart is shown in Figure.3.

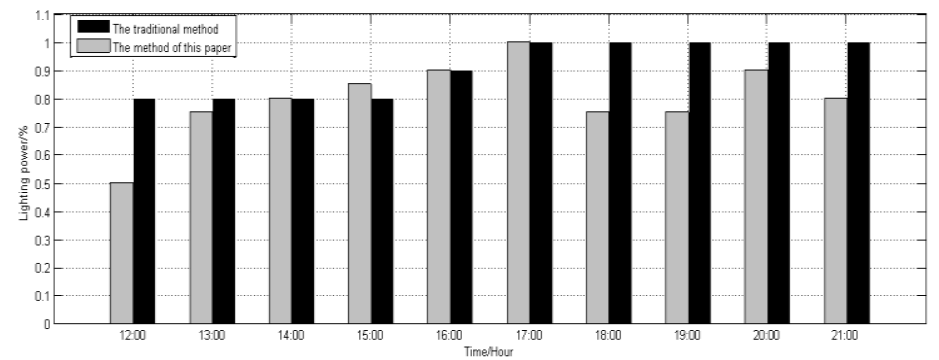

Fig.3. Illumination intensity output contrast chart

\section{Conclusion}

Based on SOM-BP neural network, the forecasting of the crowd flow improves predictive accuracy. On this basis, the flow data predicted is used in energyefficient lighting combined with the lighting intensity output, the method mentioned in the paper has realized energy-saving, has provided the new feasible plan for the energy conservation illumination.

Crowd flow forecast results have certain spots of high error, because this 
method does not take the effects of weather or other human factors into account. How to make the model more accurate, this is the problem to be studied.

\section{Acknowledgement}

This work is supported by Hu-Jiang Fund (C14002) and Shanghai Zhang-Jiang national key projects of independent innovation (201310-PI-B2-008).

\section{References}

1. Yun Sun, Ming-Tao Li, Xiao-Hui Yao. Realization of crowd flow forecast based on BP neural network [J]. Journal of Safety Science and Technology, 2010(6): 61-65.

2. Yan-Hua Wang. Some new progress in the study of SOM [J]. FuJian Computer, 2013(11):1-4.

3. Jin-Hua Yan. Based on SOM neural network load characteristic classification [J]. Popular Science, 2013, 15(172):31-33.

4. Jian-Wu Liang, Zu-Quan Chen, Tan Hai-Long. Short-term load forecasting combination of clustering and support vector machine (SVM) method [J]. Electrical power system and automated journal, 2011, 23(1):34-38.

5. Arroyo J R.Espinola. Different approaches to forecast interval time series: a comparison in finance [J]. Computational Economics, 2011, 37(2): 169-191.

6. Jun-Song Wang. Based on Elman neural network current capacity and forecast [J]. Computer engineering, 2009, 35(9): 190-191.

7. Li-Xin Ma, Yi Jing-Jing, Zhen Xiao-Dong etc. Research on power load forecasting method in intelligent electrical environment [J]. Mechanical and electrical engineering, 2015, 32(9):1233-1237.

8. Li-Xin Ma, Yuan Li. Sector power load characteristic extraction and forecast method [J]. Control engineering, 2015, 22(4): 645-648.

9. Feng Shi, Xiao-Chuan Wang, Yu Lei etc. MATLAB neural network 30 case analyses [M]. Beijing: Beijing University of aeronautics and astronautics press, 2010.

10. Morad R.Atif, Anca D.Galasiu. Energy performance of daylight-linked automatic lighting control systems in large atrium spaces: report on two field-monitored case studies [J]. Energy and Buildings. July, 2003. 